\title{
Some remarks on the bias distribution analysis of discrete-time identification algorithms based on pseudo-linear regressions
}

\author{
Bernard Vau, Henri Bourlès \\ SATIE, Ecole normale supérieure Paris-Saclay 94230 Cachan France
}

March 22, 2021

\begin{abstract}
In 1998, A. Karimi and I.D. Landau published in the journal "Systems and Control letters" an article entitled "Comparison of the closed-loop identification methods in terms of bias distribution". One of its main purposes was to provide a bias distribution analysis in the frequency domain of closed-loop output error identification algorithms that had been recently developed. The expressions provided in that paper are only valid for prediction error identification methods (PEM), not for pseudo-linear regression (PLR) ones, for which we give the correct frequency domain bias analysis, both in open- and closed-loop. Although PLR was initially (and is still) considered as an approximation of PEM, we show that it gives better results at high frequencies.
\end{abstract}

\section{Introduction}

In the field of discrete-time identification, the bias distribution analysis over frequency domain is very powerful in order to evaluate the influence of input and noise spectra on an identified model, and to assess qualitatively the model that can be obtained if it has not the same structure as the identified system. This method was first introduced in [9] for open-loop identification; see [7] for more details, especially in the context of closed-loop operations. In these two references, this bias analysis has been developed in the perspective of prediction error methods (PEM), which aim at minimizing a one step further prediction error variance. In 4 and 5 , Karimi and Landau used the same method to infer the bias distribution of closed-loop algorithms. In [4] (section 5) and [6] (section 6 ), it is claimed that this analysis is valid for CLOE, F-CLOE and AF-CLOE algorithms which are of PLR type. However, this is not true as already noticed in [5], p.308, although PLR was initially (and is still) considered as an approximation of PEM (see, e.g., remark 8.4.2 of 3); rather, PLR algorithms tend to cancel the correlation function between the prediction error and an observation vector, which in general is the regressor of the predictor, possibly filtered (see the correlation approach developed by Ljung in [7). If the system dynamics is approximately known beforehand, the difference between PEM and PLR can be made quite small, as shown in section 4, with an appropriate regressor filtering. But this approach presupposes that what one is seeking is already known, a vicious circle which prevents from addressing the core of the problem. It is said in [5] (p. 308) that the bias distribution cannot be computed for CLOE algorithm. Therefore, bias

*bernard.vau@satie.ens-cachan.fr 
distribution of PLR algorithms, such as CLOE, is an open problem, which is solved here; this bias distribution is determined and we show that it is quite different from that of PEM. To do this we introduce in section 3, the concept of equivalent prediction error most of the time a non-measurable signal - whose variance is effectively minimized by the PLR algorithm, even if the identified system is not in the model set. This approach shows that compared to PEM, PLR schemes strongly penalize the model misfit at high frequency, in a way comparable to the classical open-loop least-squares algorithm, whatever the predictor model is (output error, ARMAX, etc.). In section 5, an example is given, that relies on the Vinnicombe gap, in order to compare the model misfit of a PEM scheme and its corresponding PLR one: It brings to light the discrepancy between the two methods in case of a closed-loop output error (CLOE) identification structure.

\section{Optimal prediction error and bias distribution anal- ysis of PEM algorithms}

At first, let us recall briefly the model structures used here, both in open-and closedloop, and the manner to obtain the bias distribution from PEM algorithms. According to Landau et al. (4], p. 44), we distinguish between equation error models and output error models. Equation error model in open-loop corresponds to the equation:

$$
A\left(q^{-1}\right) y(t)=B\left(q^{-1}\right) u(t)+C\left(q^{-1}\right) e(t)
$$

with $G\left(q^{-1}\right)=\frac{B\left(q^{-1}\right)}{A\left(q^{-1}\right)}$, and $W\left(q^{-1}\right)=\frac{C\left(q^{-1}\right)}{A\left(q^{-1}\right)}$, respectively the deterministic and stochastic parts of the model; $q^{-1}$ the shift backward operator, $A\left(q^{-1}\right)$ is a monic polynomial, $W\left(q^{-1}\right)$ is the ratio of two monic polynomials, and $u(t), y(t), e(t)$ are the input, the output, and a centered gaussian white noise, respectively.

According to the noise structure, we distinguish between the following cases:

- when $C\left(q^{-1}\right)=1$, corresponding to the ARX model,

- when $C\left(q^{-1}\right)$ is a monic polynomial of degree strictly greater than 0 , corresponding to the ARMAX model.

Other equation error models exist (e.g. ARARMAX, etc.) but they are not treated in this paper. On the other hand, the output error model in open-loop is given by

$$
y(t)=G\left(q^{-1}\right) u(t)+v(t)
$$

where $v(t)$ is a centered gaussian noise not necessarily white, but uncorrelated with the input.

Let us call $\widehat{G}\left(q^{-1}\right)$ and $\widehat{W}\left(q^{-1}\right)$ the estimations of $G\left(q^{-1}\right)$ and $W\left(q^{-1}\right)$, respectively. In the case of an open-loop equation error model, the prediction error is given by (5, $(3.3)),([5,(9.62)$ :

$$
\varepsilon(t)=\widehat{W}\left(q^{-1}\right)\left\{\left(G\left(q^{-1}\right)-\widehat{G}\left(q^{-1}\right)\right) u(t)+\left(W\left(q^{-1}\right)-\widehat{W}\left(q^{-1}\right)\right) e(t)\right\}+e(t)
$$

whereas the optimal error for the open-loop output error model is simply:

$$
\varepsilon(t)=\left(G\left(q^{-1}\right)-\widehat{G}\left(q^{-1}\right)\right) u(t)+v(t)
$$

The closed-loop case is more complicated, due to the feedback control law. We assume that the controller has an R-S structure, i.e. $S\left(q^{-1}\right) u(t)=-R\left(q^{-1}\right) y(t)$; let us define the direct sensitivity function (transfer function from the output noise to the output):

$$
S_{y p}\left(q^{-1}\right)=\frac{A\left(q^{-1}\right) S\left(q^{-1}\right)}{A\left(q^{-1}\right) S\left(q^{-1}\right)+B\left(q^{-1}\right) R\left(q^{-1}\right)}
$$


In the context of an equation error model, in which the model input is given by $S\left(q^{-1}\right) \widehat{u}(t)=$ $-R\left(q^{-1}\right) \widehat{y}(t)$, the optimal predicted output is

$$
\widehat{y}(t)=\widehat{G} \widehat{u}(t)+\widehat{W}\left(q^{-1}\right) \varepsilon(t)
$$

where $\widehat{\bar{W}}\left(q^{-1}\right)=\widehat{W}\left(q^{-1}\right)-\widehat{S}_{y p}^{-1}\left(q^{-1}\right)$ (see [4], eq. (5.7) sq.), thus we have:

$$
\varepsilon(t)=\widehat{W}^{-1}\left(q^{-1}\right)\left\{(G-\widehat{G}) S_{y p} r_{u}(t)+\left(W S_{y p} \widehat{S}_{y p}^{-1}-\widehat{W}\right) e(t)\right\}+e(t)
$$

This expression is directly obtained from $(4,(5.12))$.

The optimal predicted output is given by $\widehat{y}(t)=\widehat{G} \widehat{u}(t)$, and the corresponding optimal prediction error of output error model by

$$
\varepsilon(t)=\widehat{S}_{y p}(G-\widehat{G}) S_{y p} r_{u}(t)+S_{y p} v(t)
$$

where $\widehat{S}_{y p}=\frac{\widehat{A} S}{\widehat{A} S+\widehat{B} R}, \widehat{A}, \widehat{B}$ being the estimations of $A$ and $B$ respectively (where the dependance in $q^{-1}$ is omitted).

The purpose of PEM algorithms is to minimize the prediction error variance $\mathbf{E}\left[\varepsilon^{2}(t)\right]$, and whatever the algorithm structure is, both in open or closed-loop, one obtains the optimal estimated parameter vector $\widehat{\theta}_{P E M}^{*}$ :

$$
\widehat{\theta}_{P E M}^{*}=\operatorname{Arg} \min \mathbf{E}\left[\varepsilon^{2}(t)\right]=\operatorname{Arg} \min \int_{-\pi}^{+\pi}\left|\mathcal{Z}\{\varepsilon\}\left(e^{i \omega}\right)\right|^{2} d \omega
$$

where $\mathcal{Z}$ is the z-transform. This expression is at the origin of bias analysis for PEM algorithms.

\section{Bias distribution of pseudo-linear regression algo- rithms}

\subsection{Equivalent prediction error}

The a posteriori model predicted output $\widehat{y}(t+1)$ is provided by $\widehat{y}(t+1)=\widehat{\theta}(t+1) \phi(t, \widehat{\theta})$, where $\phi(t, \widehat{\theta})$ is the regressor structure, generally depending on $\widehat{\theta}$. The a posteriori prediction error is given by the expression: $\varepsilon(t+1)=y(t+1)-\widehat{y}(t+1, \widehat{\theta})$. Most of the PLR identification procedures are solved recursively with the so-called parameter adaptation algorithm (PAA):

$$
\begin{aligned}
\widehat{\theta}(t+1) & =\widehat{\theta}(t)+F(t) \phi(t) \varepsilon(t+1) \\
F^{-1}(t+1) & =\lambda_{1} F^{-1}(t)+\lambda_{2} \phi(t) \phi^{T}(t)
\end{aligned}
$$

$F(t)$ is the adaptation gain (positive definite matrix), $0<\lambda_{1} \leq 1,0 \leq \lambda_{2}<2$ are forgetting factors. The stationary condition of the PAA is (see [7], p. 224):

$$
\mathbf{E}[\varepsilon(t+1) \phi(t)]=0
$$

Lemma 1. In general, the stationarity condition of the parameter adaptation algorithm $\mathbf{E}[\varepsilon(t+1) \phi(t, \widehat{\theta})]=0$, is not the one associated with the prediction error variance $\mathbf{E}\left[\varepsilon^{2}(t)\right]$ minimization. 
Proof. As a counterexample let us consider the extended least squares algorithm (corresponding to an ARMAX model), for which the predicted output is $\widehat{y}(t+1)=\widehat{\theta}^{T} \phi(t)$. Let

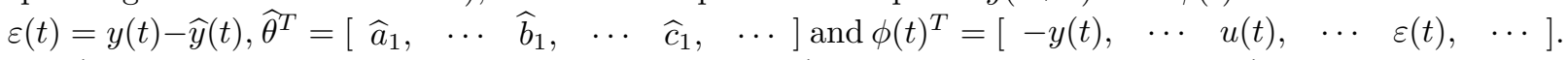
Let $\widehat{C}\left(q^{-1}\right)=1+\widehat{c}_{1} q^{-1}+\widehat{c}_{2} q^{-2} \cdots$ and assume that $\widehat{C}\left(q^{-1}\right) \neq 1$. One has that $\phi(t, \widehat{\theta})=$ $-\widehat{C}\left(q^{-1}, \widehat{\theta}\right) \frac{\partial \varepsilon(t+1)}{\partial \widehat{\theta}}$. In this case the stationary condition of the parameter adaptation algorithm is $\mathbf{E}\left[\varepsilon(t+1) \widehat{C}\left(q^{-1}\right) \frac{\partial \varepsilon(t+1)}{\partial \widehat{\theta}}\right]=0$. Therefore $\frac{\partial}{\partial \widehat{\theta}} \mathbf{E}\left[\varepsilon(t+1)^{2}\right]=2 \mathbf{E}\left[\varepsilon(t+1) \frac{\partial \varepsilon(t+1)}{\partial \widehat{\theta}}\right]$ cannot be zero unless $\{\varepsilon(t+1)\}$ is white, and that cannot happen if the system is not in the model set.

Lemma 2. In PLR schemes, the stationarity condition $\mathbf{E}[\varepsilon(t+1) \phi(t, \hat{\theta})]=0$ is the stationary condition of the variance minimization problem of the signal $\varepsilon_{E}(t+1, \widehat{\theta})$, called the "equivalent prediction error" in the sequel, and characterized by the following two conditions:

1) If the system is in the model set and if the estimated parameter vector $\widehat{\theta}_{P L R}^{*}$ is equal to the true parameters vector $\theta\left(\theta=\widehat{\theta}_{P L R}^{*}\right)$, then one has:

- for the equation error model: $\varepsilon_{E}\left(t+1, \widehat{\theta}_{P L R}^{*}\right)=\varepsilon_{E}(t+1, \theta)=\varepsilon(t+1, \theta)=e(t+1)$

- for the open-loop output error model: $\varepsilon_{E}\left(t+1, \widehat{\theta}_{P L R}^{*}\right)=\varepsilon_{E}(t+1, \theta)=\varepsilon(t+1, \theta)=v(t+1)$

- for the closed-loop output error model: $\varepsilon_{E}\left(t+1, \widehat{\theta}_{P L R}^{*}\right)=\varepsilon_{E}(t+1, \theta)=\varepsilon(t+1, \theta)=S_{y p} v(t+1)$

2) The vector $\phi_{E}(t)=-\frac{\partial \varepsilon_{E}(t+1)}{\partial \widehat{\theta}}$, called the "equivalent regressor", is not a function of $\widehat{\theta}$, i.e $\frac{\partial \phi_{E}(t)}{\partial \widehat{\theta}}=0$.

Proof. By 2), $\frac{\partial \phi_{E}(t)}{\partial \widehat{\theta}}=0$, so that $\varepsilon_{E}(t+1)=-\widehat{\theta}^{T} \phi_{E}(t)+k(k$ independant of $\widehat{\theta})$. By 1$)$ we get:

$e(t+1)=-\theta^{T} \phi_{E}(t)+k$ for the equation error model, $v(t+1)=-\theta^{T} \phi_{E}(t)+k$ for the open-loop output error model, $S_{y p} v(t+1)=-\theta^{T} \phi_{E}(t)+k$ for the closed-loop output error model.

Combining the preceding equations, one gets the following prediction error expressions: $\varepsilon_{E}(t+1)=(\theta-\widehat{\theta})^{T} \phi_{E}(t)+e(t+1)$ for the equation error model, $\varepsilon_{E}(t+1)=(\theta-\widehat{\theta})^{T} \phi_{E}(t)+v(t+1)$ for the open-loop output error model, $\varepsilon_{E}(t+1)=(\theta-\widehat{\theta})^{T} \phi_{E}(t)+S_{y p} v(t+1)$ for the closed-loop output error model.

The stationarity condition applied to these equations is $\mathbf{E}\left[\varepsilon_{E}(t+1) \phi_{E}(t)\right]=0$. Since $\phi_{E}(t)=-\frac{\partial \varepsilon_{E}(t+1)}{\partial \widehat{\theta}}$, this stationnarity condition can be rewritten $\mathbf{E}\left[\varepsilon_{E}(t+1) \frac{\partial \varepsilon_{E}}{\partial \widehat{\theta}}\right]=0$, which is the gradient of $\mathbf{E}\left[\varepsilon_{E}^{2}(t+1)\right]$ with respect to $\widehat{\theta}$.

Lemma 3. One has $\widehat{\theta}_{P L R}^{*}=\operatorname{Arg} \min \int_{-\pi}^{\pi} \mid \mathcal{Z}\left\{\varepsilon_{E}\right\}\left(e^{i \omega}\right) \|^{2} d \omega$.

Proof. The minimization problem of $\mathbf{E}\left[\varepsilon_{E}^{2}(t)\right]$ is convex since $\varepsilon_{E}(t)$ is expressed linearly in function of $\phi_{E}(t)$, as shown by the proof of lemma 2 .

In this paper, we assume that the dependence of $\widehat{y}(t+1)$ with respect to $\widehat{\theta}$ can be expressed uniquely via $\varepsilon(t, \widehat{\theta})$, which is the case of the ARMAX and output error predictors (in open- and closed-loop operations), as shown in Table1. Under this assumption, we can write: $\frac{\partial \varepsilon(t+1)}{\partial \widehat{\theta}}=\frac{-\partial \widehat{y}(t+1)}{\partial \widehat{\theta}}=-\phi(t)-\widehat{\theta}^{T} \frac{\partial \phi}{\partial(q \varepsilon)} \frac{\partial \varepsilon(t+1)}{\partial \widehat{\theta}}$. Let us define $Q\left(q^{-1}, \widehat{\theta}\right)=1+\widehat{\theta}^{T} \frac{\partial \phi}{\partial(q \varepsilon)}$, 
so that $Q\left(q^{-1}, \widehat{\theta}\right) \frac{\partial \varepsilon(t+1)}{\partial \widehat{\theta}}=-\phi(t)$. The computation of $\frac{\partial \phi}{\partial(q \varepsilon)}$ can be performed using the remarks in 6 . We can now state the main result of this section.

Theorem 1. Let $Q\left(q^{-1}, \widehat{\theta}\right)=1+\widehat{\theta}^{T} \frac{\partial \phi}{\partial(q \varepsilon)}$. Assuming that $\frac{\partial^{2} \phi}{\partial(q \varepsilon) \partial \widehat{\theta}}=0$, the equivalent prediction error defined in lemma 2 is such that:

- for the equation error model:

$$
\varepsilon_{E}(t+1)=Q\left(q^{-1}, \widehat{\theta}\right) \varepsilon(t+1)+\left(1-Q\left(q^{-1}, \widehat{\theta}\right)\right) e(t+1)
$$

- for the open-loop output error model:

$$
\varepsilon_{E}(t+1)=Q\left(q^{-1}, \widehat{\theta}\right) \varepsilon(t+1)+\left(1-Q\left(q^{-1}, \widehat{\theta}\right)\right) v(t+1)
$$

- for the closed-loop output error model:

$$
\varepsilon_{E}(t+1)=Q\left(q^{-1}, \widehat{\theta}\right) \varepsilon(t+1)+\left(1-Q\left(q^{-1}, \widehat{\theta}\right)\right) S_{y p}\left(q^{-1}\right) v(t+1)
$$

Proof. One has to verify Conditions 1) and 2) of Lemma 2. 1): If the system is in the model set and $\theta=\widehat{\theta}$, it is immediate to check that:

$\varepsilon_{E}(t+1)=e(t+1)$ for the equation error model,

$\varepsilon_{E}(t+1)=v(t+1)$ for open-loop output error model,

$\varepsilon_{E}(t+1)=S_{y p}\left(q^{-1}\right) v(t+1)$ for closed-loop output error model.

$2)$ : One has to verify that $\phi_{E}(t)=\frac{-\partial \varepsilon_{E}(t+1)}{\partial \widehat{\theta}}$ is not a function of $\widehat{\theta}$, i.e. $\frac{\partial \phi_{E}(t)}{\partial \widehat{\theta}}=0$. For this purpose let us compute:

$\frac{\partial \varepsilon_{E}(t+1)}{\partial \widehat{\theta}}=Q \frac{\partial \varepsilon(t+1)}{\partial \widehat{\theta}}+\frac{\partial Q}{\partial \widehat{\theta}}(\varepsilon(t+1)-e(t+1))=-\phi(t)+\frac{\partial Q}{\partial \widehat{\theta}}(\varepsilon(t+1)-e(t+1))$ for the equation error model. But $\frac{\partial Q}{\partial \widehat{\theta}}=\frac{\partial \phi}{\partial(q \varepsilon)}+\widehat{\theta}^{T} \frac{\partial^{2} \phi}{\partial(q \varepsilon) \partial \widehat{\theta}}$. Under the assumption $\frac{\partial^{2} \phi}{\partial(q \varepsilon) \partial \widehat{\theta}}=0$, we obtain $\frac{\partial Q}{\partial \widehat{\theta}}=\frac{\partial \phi}{\partial(q \varepsilon)}$. As $\phi_{E}(t)=\frac{-\partial \varepsilon_{E}(t+1)}{\partial \widehat{\theta}}$, one gets $\frac{\partial \phi_{E}(t)}{\partial \widehat{\theta}}=-\frac{\partial \phi(t)}{\partial \widehat{\theta}}+\frac{\partial \phi(t)}{\partial \widehat{\theta}}=0$. The same result holds for output error models (both in open- and closed-loop), substituting $e(t+1)$ by $v(t+1)$ and by $S_{y p}\left(q^{-1}\right) v(t+1)$ respectively.

\subsection{Expression of the bias distribution of PLR algorithms}

\begin{tabular}{|c|c|c|}
\hline \multicolumn{2}{|c|}{$\begin{array}{c}\text { NOISE } \\
\text { MODEL }\end{array}$} & PREDICTED OUTPUT $\widehat{y}(t+1)$ and REGRESSOR $\phi(t)$ \\
\hline \multirow{6}{*}{ 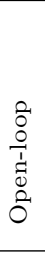 } & \multirow{2}{*}{ ARX } & $\widehat{y}(t+1)=-\widehat{a}_{1} y(t)-\widehat{a}_{2} y(t-1)+\cdots+\widehat{b}_{1} u(t)+\widehat{b}_{2} u(t-1)+\cdots$ \\
\hline & & $\phi(t)=[-y(t),-y(t-1), \cdots u(t), u(t-1), \cdots]$ \\
\hline & \multirow{2}{*}{ ARMAX } & $\widehat{y}(t+1)=-\widehat{a}_{1} y(t)-\widehat{a}_{2} y(t-1)+\cdots+\widehat{b}_{1} u(t)+\widehat{b}_{2} u(t-1)+\cdots+\widehat{c}_{1} \varepsilon(t)+\widehat{c}_{2} \varepsilon(t-1)+\cdots$ \\
\hline & & $\phi(t)=[-y(t),-y(t-1), \cdots u(t), u(t-1), \cdots \varepsilon(t), \varepsilon(t-1), \cdots]$ \\
\hline & \multirow{2}{*}{$\begin{array}{c}\text { OUTPUT } \\
\text { ERROR }\end{array}$} & $\widehat{y}(t+1)=-\widehat{a}_{1} \widehat{y}(t)-\widehat{a}_{2} \widehat{y}(t-1)+\cdots+\widehat{b}_{1} u(t)+\widehat{b}_{2} u(t-1)+\cdots$ \\
\hline & & $\phi(t)=[-\widehat{y}(t),-\widehat{y}(t-1), \cdots u(t), u(t-1), \cdots]$ \\
\hline \multirow{4}{*}{$\begin{array}{l}0 \\
0 \\
\frac{0}{1} \\
\frac{1}{d} \\
0 \\
0 \\
0 \\
0\end{array}$} & \multirow{2}{*}{ ARMAX } & $\widehat{y}(t+1)=-\widehat{a}_{1} \widehat{y}(t)-\widehat{a}_{2} \widehat{y}(t-1)+\cdots+\widehat{b}_{1} \widehat{u}(t)+\widehat{b}_{2} \widehat{u}(t-1)+\cdots+\widehat{h}_{1} \varepsilon(t)+\widehat{h}_{2} \varepsilon(t-1)+\cdots$ \\
\hline & & $\phi(t)=[-\widehat{y}(t),-\widehat{y}(t-1), \cdots \widehat{u}(t), \widehat{u}(t-1), \cdots \varepsilon(t), \varepsilon(t-1), \cdots]$ \\
\hline & \multirow{2}{*}{$\begin{array}{c}\text { OUTPUT } \\
\text { ERROR }\end{array}$} & $\widehat{y}(t+1)=-\widehat{a}_{1} \widehat{y}(t)-\widehat{a}_{2} \widehat{y}(t-1)+\cdots+\widehat{b}_{1} \widehat{u}(t)+\widehat{b}_{2} \widehat{u}(t-1)+\cdots$ \\
\hline & & $\phi(t)=[-\widehat{y}(t),-\widehat{y}(t-1), \cdots \widehat{u}(t), \widehat{u}(t-1), \cdots]$ \\
\hline
\end{tabular}

For each noise model, either in open- or closed-loop operations, the corresponding PLR algorithm has a specific predictor. We give in Table 1 these expressions.

Table 1: Predicted output in open- and closed-loop operations 
(Table 2 provides the expressions of $Q\left(q^{-1}, \widehat{\theta}\right)$ and $\varepsilon_{E}(t+1)$ for all predictor models presented above where $\widehat{P}=\widehat{A} S+\widehat{B} R$ ).

\begin{tabular}{|c|c|c|c|}
\hline \multicolumn{2}{|c|}{$\begin{array}{c}\text { NOISE } \\
\text { MODEL }\end{array}$} & $Q(\widehat{\theta})$ & $\varepsilon_{E}(t)$ \\
\hline \multirow{4}{*}{ Open- loop } & ARX & 1 & $\widehat{A}(G-\widehat{G}) u(t)+\widehat{A}\left(W-\frac{1}{\widehat{A}}\right) e(t)+e(t)$ \\
\cline { 2 - 4 } & ARMAX & $\widehat{C}\left(q^{-1}, \widehat{\theta}\right)$ & $\widehat{A}(G-\widehat{G}) u(t)+\widehat{A}\left(W-\frac{\widehat{C}}{\widehat{A}}\right) e(t)+e(t)$ \\
\cline { 2 - 4 } & $\begin{array}{c}\text { OUTPUT } \\
\text { ERROR }\end{array}$ & $\widehat{A}\left(q^{-1}, \widehat{\theta}\right)$ & $\widehat{A}(G-\widehat{G}) u(t)+v(t)$ \\
\hline \multirow{3}{*}{ Closed- loop } & ARMAX & $\widehat{C}\left(q^{-1}, \widehat{\theta}\right)$ & $\widehat{A}(G-\widehat{G}) S_{y p} r_{u}(t)+\widehat{A}\left(W S_{y p} \widehat{S}_{y p}^{-1}-\frac{\widehat{C}}{\widehat{A}}\right) e(t)+e(t)$ \\
\cline { 2 - 4 } & $\begin{array}{c}\text { OUTPUT } \\
\text { ERROR }\end{array}$ & $\frac{\widehat{P}\left(q^{-1}, \widehat{\theta}\right)}{S\left(q^{-1}\right)}$ & $\widehat{A}(G-\widehat{G}) S_{y p}\left(q^{-1}\right) r_{u}(t)+S_{y p}\left(q^{-1}\right) v(t)$ \\
\hline
\end{tabular}

Table 2: Expression of the equivalent prediction error (open-loop and closed-loop)

These expressions differ from those of PEM that can be computed from (2). In particular one can notice that for ARMAX and OE models, both in open- and closed-loop, the deterministic part of $\varepsilon_{E}(t)$ is weighted by $\widehat{A}$ which is the same weighting function as for the ARX model. This function strongly penalizes the high frequency misfit. Table 3 gives the expressions of the asymptotic values of the estimated parameters $\widehat{\theta}_{P L R}^{*}$ in the frequency domain. The expressions $\Phi_{u u}(\omega), \Phi_{r_{u} r_{u}}(\omega), \Phi_{e e}(\omega)$, correspond to the spectra of $u, r_{u}$ (the additive excitation on the input in closed-loop), and $e$ respectively.

\begin{tabular}{|c|c|c|}
\hline \multicolumn{2}{|c|}{ NOISE MODEL } & $\widehat{\theta}_{P L R}^{*}$ \\
\hline \multirow{3}{*}{$\begin{array}{l}\stackrel{2}{0} \\
\stackrel{0}{0} \\
\frac{1}{0} \\
0 \\
0 \\
0\end{array}$} & ARX & $\begin{array}{c}\operatorname{Argmin} \int_{-\pi}^{+\pi}\left\{\left|\widehat{A}\left(e^{i \omega}\right)\right|^{2}\left(\left|G\left(e^{i \omega}\right)-\widehat{G}\left(e^{i \omega}\right)\right|^{2} \Phi_{u u}(\omega)+\right.\right. \\
\left.\left.\ldots\left|W\left(e^{i \omega}\right)-\frac{1}{\widehat{A}\left(e^{i \omega}\right)}\right|^{2} \Phi_{e e}(\omega)\right)\right\} \mathrm{d} \omega\end{array}$ \\
\hline & ARMAX & $\begin{array}{c}\operatorname{Argmin} \int_{-\pi}^{+\pi}\left\{\left|\widehat{A}\left(e^{i \omega}\right)\right|^{2}\left(\left|G\left(e^{i \omega}\right)-\widehat{G}\left(e^{i \omega}\right)\right|^{2} \Phi_{u u}(\omega)+\right.\right. \\
\left.\left.\ldots\left|W\left(e^{i \omega}\right)-\frac{\widehat{C}}{\widehat{A}\left(e^{i \omega}\right)}\right|^{2} \Phi_{e e}(\omega)\right)\right\} \mathrm{d} \omega\end{array}$ \\
\hline & OUTPUT ERROR & $\operatorname{Argmin} \int^{+\pi}\left|\widehat{A}\left(e^{i \omega}\right)\right|^{2}\left|G\left(e^{i \omega}\right)-\widehat{G}\left(e^{i \omega}\right)\right|^{2} \Phi_{u u}(\omega) \mathrm{d} \omega$ \\
\hline \multirow{2}{*}{$\begin{array}{l}\overbrace{0}^{2} \\
\frac{1}{1} \\
0 \\
0 \\
0 \\
0 \\
0 \\
0 \\
0\end{array}$} & ARMAX & $\begin{array}{c}\operatorname{Argmin} \int_{-\pi}^{+\pi}\left\{\left|\widehat{A}\left(e^{i \omega}\right)\right|^{2}\left(\left|G\left(e^{i \omega}\right)-\widehat{G}\left(e^{i \omega}\right)\right|^{2}\left|S_{y p}\left(e^{i \omega}\right)\right|^{2} \Phi_{r_{u} r_{u}}(\omega)+\right.\right. \\
\left.\left.\ldots\left|W\left(e^{i \omega}\right) \frac{S_{y p}\left(e^{i \omega}\right)}{\widehat{S}_{y p}^{-1}\left(e^{i \omega}\right)}-\frac{\widehat{C}\left(e^{i \omega}\right)}{\widehat{A}\left(e^{i \omega}\right)}\right|^{2} \Phi_{e e}(\omega)\right)\right\} \mathrm{d} \omega\end{array}$ \\
\hline & OUTPUT ERROR & $\operatorname{Argmin} \int_{-\pi}^{+\pi}\left|\widehat{A}\left(e^{i \omega}\right)\right|^{2}\left|G\left(e^{i \omega}\right)-\widehat{G}\left(e^{i \omega}\right)\right|^{2}\left|S_{y p}\left(e^{i \omega}\right)\right|^{2} \Phi_{r_{u} r_{u}}(\omega) \mathrm{d} \omega$ \\
\hline
\end{tabular}

Table 3: Bias distribution for PLR algorithms in open- and closed-loop

By comparison, we recall in Table 4 the expressions for the estimated parameters in case of PEM methods. The expressions corresponding to ARMAX and output error models result directly from (4.186) and (4.193) of [5] in an open-loop context, and from (9.81) and (9.79) of the same book in closed-loop operation. 


\begin{tabular}{|c|c|c|}
\hline \multicolumn{2}{|c|}{ NOISE MODEL } & $\widehat{\theta}_{P E M}^{*}$ \\
\hline \multirow{3}{*}{ 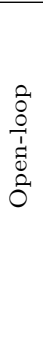 } & ARX & $\begin{array}{c}\operatorname{Argmin} \int_{-\pi}^{+\pi}\left\{\left|\widehat{A}\left(e^{i \omega}\right)\right|^{2}\left(\left|G\left(e^{i \omega}\right)-\widehat{G}\left(e^{i \omega}\right)\right|^{2} \Phi_{u u}(\omega)+\right.\right. \\
\left.\left.\ldots\left|W\left(e^{i \omega}\right)-\frac{1}{\widehat{A}\left(e^{i \omega}\right)}\right|^{2} \Phi_{e e}(\omega)\right)\right\} \mathrm{d} \omega\end{array}$ \\
\hline & ARMAX & $\begin{array}{c}\operatorname{Argmin} \int_{-\pi}^{+\pi}\left\{\left|\frac{\widehat{A}\left(e^{i \omega}\right)}{\widehat{C}\left(e^{i \omega}\right)}\right|^{2}\left(\left|G\left(e^{i \omega}\right)-\widehat{G}\left(e^{i \omega}\right)\right|^{2} \Phi_{u u}(\omega)+\right.\right. \\
\left.\left.\ldots\left|W\left(e^{i \omega}\right)-\frac{\widehat{C}}{\widehat{A}\left(e^{i \omega}\right)}\right|^{2} \Phi_{e e}(\omega)\right)\right\} \mathrm{d} \omega\end{array}$ \\
\hline & OUTPUT ERROR & $\operatorname{Argmin} \int_{-\pi}^{+\pi}\left|G\left(e^{i \omega}\right)-\widehat{G}\left(e^{i \omega}\right)\right|^{2} \Phi_{u u}(\omega) \mathrm{d} \omega$ \\
\hline \multirow{2}{*}{$\begin{array}{l}0 \\
0 \\
\frac{0}{1} \\
\frac{1}{0} \\
0 \\
0 \\
0 \\
0 \\
0\end{array}$} & ARMAX & $\begin{aligned} \operatorname{Argmin} \int_{-\pi}^{+\pi}\left\{\left|\frac{\widehat{A}\left(e^{i \omega}\right)}{\widehat{C}\left(e^{i \omega}\right)}\right|^{2}\left(\left|G\left(e^{i \omega}\right)-\widehat{G}\left(e^{i \omega}\right)\right|^{2}\left|S_{y p}\left(e^{i \omega}\right)\right|^{2} \Phi_{r_{u} r_{u}}(\omega)+\right.\right. \\
\left.\left.\ldots\left|W\left(e^{i \omega}\right) \frac{\widehat{S}_{y p}\left(e^{i \omega}\right)}{S_{y p}^{-1}\left(e^{i \omega}\right)}-\frac{\widehat{C}\left(e^{i \omega}\right)}{\widehat{A}\left(e^{i \omega}\right)}\right|^{2} \Phi_{e e}(\omega)\right)\right\} \mathrm{d} \omega\end{aligned}$ \\
\hline & OUTPUT ERROR & $\operatorname{Argmin} \int_{-\pi}^{+\pi}\left|\widehat{S}_{y p}\left(e^{i \omega}\right)\right|^{2}\left|G\left(e^{i \omega}\right)-\widehat{G}\left(e^{i \omega}\right)\right|^{2}\left|S_{y p}\left(e^{i \omega}\right)\right|^{2} \Phi_{r_{u} r_{u}}(\omega) \mathrm{d} \omega$ \\
\hline
\end{tabular}

Table 4: Bias distribution for PEM algorithms in open- and closed-loop

\section{Bias distribution for PLR algorithms with regressor filtering}

\subsection{Equivalent prediction error expressions}

In classical PLR algorithms, such as those presented in section 3 , the parameter adaptation algorithm (PAA) is fed with the a posteriori prediction error $\varepsilon(t+1)$ and the regressor $\phi(t)$. In some algorithms, a filtering is operated on the regressor such that:

$$
\phi_{f}(t)=\frac{1}{Q_{f}\left(q^{-1}\right)} \phi(t)
$$

and $\phi_{f}(t)$ is used in the PAA instead of $\phi(t) . Q_{f}\left(q^{-1}\right)$ is a ratio of monic polynomials, and we assume that $Q_{f}\left(q^{-1}\right)$ does not depend on $\widehat{\theta}$. The purpose of this filtering is usually to relax the convergence conditions, see for example (5] p. 161). With this filtering the PAA becomes (the predicted output expression is not modified):

$$
\begin{aligned}
\widehat{\theta}(t+1) & =\widehat{\theta}(t)+F(t) \phi_{f}(t) \varepsilon(t+1) \\
F^{-1}(t+1) & =\lambda_{1} F^{-1}(t)+\lambda_{2} \phi_{f}(t) \phi_{f}^{T}(t)
\end{aligned}
$$

Theorem 2. In the case of regressor filtering in the PAA, corresponding to equations (5), 6b. let $Q\left(q^{-1}, \widehat{\theta}\right)=1+\widehat{\theta}^{T} \frac{\partial \phi}{\partial(q \varepsilon)}$, and assume $\frac{\partial^{2} \phi}{\partial(q \varepsilon) \partial \widehat{\theta}}=0$. The equivalent prediction error $\varepsilon_{E}(t+1)$, as defined in proposition 2, is given by:

- for the equation error model:

$$
\varepsilon_{E}(t+1)=\frac{Q\left(q^{-1}, \widehat{\theta}\right)}{Q_{f}\left(q^{-1}, \widehat{\theta}\right)} \varepsilon(t+1)+\left(1-\frac{Q\left(q^{-1}, \widehat{\theta}\right)}{Q_{f}\left(q^{-1}, \widehat{\theta}\right)}\right) e(t+1)
$$

- for the open-loop output error model:

$$
\varepsilon_{E}(t+1)=\frac{Q\left(q^{-1}, \widehat{\theta}\right)}{Q_{f}\left(q^{-1}, \widehat{\theta}\right)} \varepsilon(t+1)+\left(1-\frac{Q\left(q^{-1}, \widehat{\theta}\right)}{Q_{f}\left(q^{-1}, \widehat{\theta}\right)}\right) v(t+1)
$$

- for the closed-loop output error model:

$$
\varepsilon_{E}(t+1)=\frac{Q\left(q^{-1}, \widehat{\theta}\right)}{Q_{f}\left(q^{-1}, \widehat{\theta}\right)} \varepsilon(t+1)+\left(1-\frac{Q\left(q^{-1}, \widehat{\theta}\right)}{Q_{f}\left(q^{-1}, \widehat{\theta}\right)}\right) S_{y p}\left(q^{-1}\right) v(t+1)
$$


Proof. We give the proof for the open-loop equation error model; it is very similar to the proof of theorem 1. Once again we check that if the system is in the model set and $\theta=\widehat{\theta}$ one has $\varepsilon_{E}(t+1)=e(t+1)$, since in this case $\varepsilon(t+1)=e(t+1)$. One must verify that $\phi_{E}(t)=\frac{-\partial \varepsilon_{E}(t+1)}{\partial \widehat{\theta}}$ is not a function of $\widehat{\theta}$, i.e. $\frac{\partial \phi_{E}(t)}{\partial \widehat{\theta}}=0$. One has

$$
\begin{aligned}
\frac{\partial \varepsilon_{E}(t+1)}{\partial \widehat{\theta}} & =\frac{Q\left(q^{-1}\right)}{Q_{f}\left(q^{-1}\right)} \frac{\partial \varepsilon(t+1)}{\partial \widehat{\theta}}+\frac{1}{Q_{f}\left(q^{-1}\right)} \frac{\partial Q}{\partial \widehat{\theta}}(\varepsilon(t+1)-e(t+1)) \\
& =-\phi_{f}(t)+\frac{1}{Q_{f}\left(q^{-1}\right)} \frac{\partial Q}{\partial \widehat{\theta}}(\varepsilon(t+1)-e(t+1)) .
\end{aligned}
$$

But $\frac{1}{Q_{f}\left(q^{-1}\right)} \frac{\partial Q}{\partial \widehat{\theta}}=\frac{\partial \phi_{f}}{\partial(q \varepsilon)}$. Therefore,

$$
\frac{\partial \varepsilon_{E}(t+1)}{\partial \widehat{\theta}}=-\phi_{f}(t)+\frac{\partial \phi_{f}}{\partial(q \varepsilon)}(\varepsilon(t+1)-e(t+1))
$$

With $\phi_{E}(t)=-\frac{\partial \varepsilon_{E}(t+1)}{\partial \widehat{\theta}}$, one obtains again $\frac{\partial \phi_{E}(t)}{\partial \widehat{\theta}}=-\frac{\partial \phi_{f}(t)}{\partial \widehat{\theta}}+\frac{\partial \phi_{f}(t)}{\partial \widehat{\theta}}=0$, assuming that $\frac{\partial^{2} \phi}{\partial(q \varepsilon) \partial \widehat{\theta}}=0$, i.e. $\frac{\partial^{2} \phi_{f}}{\partial(q \varepsilon) \partial \widehat{\theta}}=0$ (as $Q_{f}\left(q^{-1}\right)$ does not depends on $\left.\widehat{\theta}\right)$. The generalization to open- and closed-loop output error schemes is immediate by substituting $e(t)$ by $v(t)$ and $S_{y p}\left(q^{-1}\right) v(t)$, respectively.

This regressor filtering has been applied in the litterature to the output error algorithms. In open loop, if $Q_{f}\left(q^{-1}\right)$ is a monic polynomial with $Q_{f}\left(q^{-1}\right)=A_{o}\left(q^{-1}\right)$, the algorithm corresponds to the so-called F-OLOE [5], p.173 of Landau et al. In closed-loop operations, if we use $Q_{f}\left(q^{-1}\right)=\frac{P_{o}\left(q^{-1}\right)}{S\left(q^{-1}\right)}$, we get Landau's F-CLOE algorithm where $P_{o}$ an estimation of the closed loop characteristic polynomial. The equivalent prediction error is provided in Table 5 and Table 6 gives the associated bias distributions.

\begin{tabular}{|c|c|c|c|}
\hline \multicolumn{2}{|c|}{ NOISE MODEL } & $\frac{Q(\widehat{\theta})}{Q_{f}(\widehat{\theta})}$ & $\varepsilon_{E}(t)$ \\
\hline $\begin{array}{c}\text { Open- } \\
\text { loop }\end{array}$ & OUTPUT ERROR & $\frac{\widehat{A}}{A_{0}}$ & $\frac{\widehat{A}}{A_{0}}(G-\widehat{G}) u(t)+v(t)$ \\
\hline $\begin{array}{c}\text { Closed- } \\
\text { loop }\end{array}$ & OUTPUT ERROR & $\frac{\widehat{P}}{P_{0}}$ & $\frac{\widehat{A} S}{P_{0}}(G-\widehat{G}) S_{y p} r_{u}(t)+S_{y p} v(t)$ \\
\hline
\end{tabular}

Table 5: Equivalent prediction error in the case of regressor filtering

In [5] p.175 and p. 300, adaptive versions of F-OLOE and F-CLOE algorithms are proposed, consisting in imposing respectively: $A_{0}\left(q^{-1}, t\right)=\widehat{A}\left(q^{-1}, t\right)$ and $P_{0}\left(q^{-1}, t\right)=$

\begin{tabular}{|c|c|c|}
\hline \multicolumn{2}{|c|}{ NOISE MODEL } & $\widehat{\theta}_{P L R}^{*}$ \\
\hline $\begin{array}{c}\text { Open- } \\
\text { loop }\end{array}$ & OUTPUT ERROR & Argmin $\int_{-\pi}^{+\pi}\left|\frac{\widehat{A}\left(e^{i \omega}\right)}{A_{0}\left(e^{i \omega}\right)}\right|^{2}\left|G\left(e^{i \omega}\right)-\widehat{G}\left(e^{i \omega}\right)\right|^{2} \Phi_{u u}(\omega) \mathrm{d} \omega$ \\
\hline $\begin{array}{c}\text { Closed- } \\
\text { loop }\end{array}$ & OUTPUT ERROR & $\operatorname{Argmin} \int_{-\pi}^{+\pi}\left|\frac{\widehat{A}\left(e^{i \omega}\right) S\left(e^{i \omega}\right)}{P_{0}\left(e^{i \omega}\right)}\right|^{2}\left|G\left(e^{i \omega}\right)-\widehat{G}\left(e^{i \omega}\right)\right|^{2} \times$ \\
$\cdots\left|S_{y p}\left(e^{i \omega}\right)\right|^{2} \Phi_{r_{u} r_{u}}(\omega) \mathrm{d} \omega$ \\
\hline
\end{tabular}

Table 6: Bias distribution in case of regressor filtering in the parameter adaptation algorithm 
$\widehat{A}\left(q^{-1}, t\right) S\left(q^{-1}\right)+\widehat{B}\left(q^{-1}, t\right) R\left(q^{-1}\right)$. We notice that, according to Table 6 , the associated bias distribution corresponds to that of PEM algorithms in Table 4 . This confirms that the so-called AF-OLOE and AF-CLOE algorithms are recursive PEM algorithms, a point which was already suggested in [5], p. 308.

\section{Simulations}

In order to show the relevance of the above analysis, we propose a set of simulations based on the same example as the one presented in (4, section 6$)$. It concerns the closed-loop identification of a system $G\left(q^{-1}\right)$ :

$$
G\left(q^{-1}\right)=\frac{q^{-1}+0.5 q^{-2}}{\left(1-1.5 q^{-1}+0.7 q^{-2}\right)\left(1-q^{-1}\right)}
$$

fed back by a controller $K\left(q^{-1}\right)$ :

$$
K\left(q^{-1}\right)=\frac{0.8659-1.2763 q^{-1}+0.5204 q^{-2}}{\left(1-q^{-1}\right)\left(1+0.3717 q^{-1}\right)}
$$

The excitation is a 9 registers PRBS introduced additively on the system input. The internal PRBS period being equal to the sampling period, the spectral power density can be considered constant over the frequency range. The model order is chosen equal to 2 , as in 4, thus a bias is necessarily present on the identified model. We compare the identification results of two models:

- the recursive PLR CLOE algorithm without prediction error filtering, method as employed in [4,

- a non recursive PEM CLOE method without prediction error filtering, by computing the sensitivity function of the prediction error with respect to the estimated parameters, and performing a non-linear optimization.

We recall the theoretical bias distribution of CLOE scheme without regressor filtering derived in this paper for PLR methods (see the last row of Table 3, respectively):

$$
\widehat{\theta}_{P L R}^{*}=\operatorname{Argmin} \int_{-\pi}^{+\pi}\left|\widehat{A}\left(e^{i \omega}\right)\right|^{2}\left|G\left(e^{i \omega}\right)-\widehat{G}\left(e^{i \omega}\right)\right|^{2}\left|S_{y p}\left(e^{i \omega}\right)\right|^{2} \Phi_{r_{u} r_{u}}(\omega) \mathrm{d} \omega
$$

and for PEM (last row of Table 4):

$$
\widehat{\theta}_{P E M}^{*}=\operatorname{Arg} \min \int_{-\pi}^{+\pi}\left|\frac{\widehat{A}\left(e^{i \omega}\right) \widehat{S}\left(e^{i \omega}\right)}{\widehat{P}\left(e^{i \omega}\right)}\right|^{2}\left|G\left(e^{i \omega}\right)-\widehat{G}\left(e^{i \omega}\right)\right|^{2}\left|S_{y p}\left(e^{i \omega}\right)\right|^{2} \Phi_{r_{u} r_{u}}(\omega) \mathrm{d} \omega
$$

where $\widehat{P}\left(q^{-1}\right)=\widehat{A}\left(q^{-1}\right) S\left(q^{-1}\right)+\widehat{B}\left(q^{-1}\right) R\left(q^{-1}\right)$.

We notice that these two expressions differ by the introduction of the term $\left|\frac{S\left(e^{i \omega}\right)}{\widehat{P}\left(e^{i \omega}\right)}\right|^{2}$ in the PEM scheme. Therefore we can expect that the PEM model fit must be better than the PLR model fit at frequencies where the filter $\frac{S\left(q^{-1}\right)}{\widehat{P}\left(q^{-1}\right)}$ magnitude is the largest, and worse at frequencies where this magnitude is small. This is exactly what is observed. Figure 1 displays the Bode diagram of the real system compared to those of the two identified models. Figure 2 top graph shows the Vinnicombe gap (see [8]) from the real system 
to these two identified models, whereas the bottom graph displays the magnitude of the filter $\frac{S\left(q^{-1}\right.}{\widehat{P}\left(q^{-1}\right.}$. PEM effectively provides a better adjustment around normalized frequencies equal to 0.15 , corresponding to the largest magnitude of the said filter. Concomitantly, we notice a significant misfit at high frequency (compared to the PLR method), and another misfit at very low frequency. The misfit at low frequency is not as important as at high frequency. It can be explained by the influence of the second weighting function $\left|S_{y p}\left(e^{i \omega}\right)\right|^{2}$ entering in the expressions (7), (8), that has a decreasing magnitude towards zero, because of an integrator in the controller.
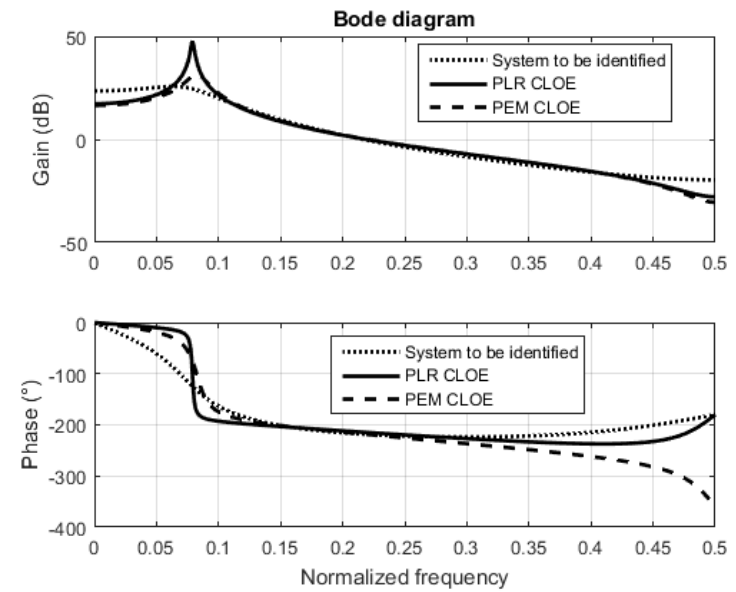

Figure 1: PLR CLOE and PEM CLOE models compared to the real system
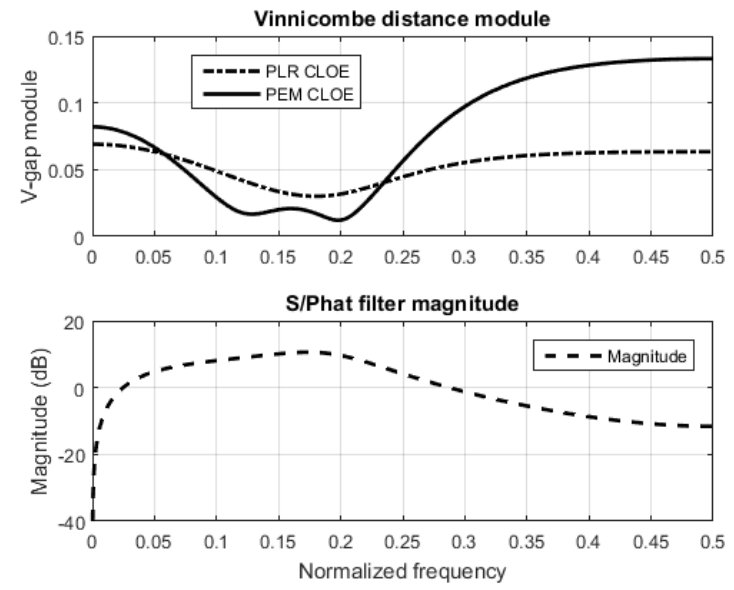

Figure 2: Vinnicombe gap from PLR CLOE and PEM CLOE models to the real system, and filter $\frac{S\left(q^{-1}\right)}{\widehat{P}\left(q^{-1}\right)}$ magnitude. 


\section{Conclusion}

We have demonstrated that the bias distribution expression in the frequency domain of pseudo-linear regression identification algorithms differs from prediction error method schemes, both in open- and closed-loop operations. In particular, we have shown that pseudo-linear regression algorithms do not minimize the prediction error variance. That led us to introduce the concept of equivalent prediction error whose variance is effectively minimized by the pseudo-linear regression schemes. We have derived a list of bias distributions for algorithms belonging to this class. These expressions show that, compared to prediction error methods, most of the pseudo-linear regression identification structures -without regressor filtering- provide a better model fit in high frequency. This filtering in the parameter adaptation algorithm induces a change in the bias distribution, therefore it can be used not only to relax the convergence conditions of algorithms, but also to enhance or depress the model accuracy over a given frequency range.

\section{Appendix}

Extended Hilbert spaces are Fréchet spaces, not Banach spaces, so that the classical differential calculus does not apply in this context. Some hints are given here on the differential calculus in extended Hilbert spaces. For the sake of simplicity, the Hilbert space considered is the space $l_{2}$ of square-summable sequences which are zero for $t<0$. The truncation operator $P_{t}$, determined for all $t \geq 0$ and any $x \in l_{2}$ by $P_{t} x(\tau)=x(\tau)$ if $t \leq \tau$, and $P_{t} x(\tau)=0$ otherwise, is an orthonormal projection of $l_{2}$, and a resolution of identity. An operator $\mathcal{G}: l_{2 e} \rightarrow l_{2 e}$ is causal if and only if $\mathcal{P}_{t} \circ \mathcal{G} \circ \mathcal{P}_{t}=\mathcal{P}_{t} \circ \mathcal{G}$ for all $t \in \mathbb{Z}_{+}$(see [2], section 1.B). As easily shown, a causal operator is continuous at $u^{0} \in l_{2 e}$ (in the usual sense, when the domain and the codomain of $G$ are both endowed with their canonical locally convex topology) if, and only if $P_{t} \circ G \circ P_{t}$, viewed as an operator from $l_{2}$ to $l_{2}$, is continuous at $u_{t}^{0}$ for all $t \in \mathbb{Z}_{+}$. Every causal linear operator is continuous, as shown by the proof of ([1, Section 7.2, Thm. 22) (mutatis mutandis).

A causal operator is called differentiable at $u_{t}^{0} \in l_{2 e}$ if $\mathcal{P}_{t} \circ \mathcal{G} \circ \mathcal{P}_{t}$ is differentiable at $u_{t}^{0}$, for all $t \in \mathbb{Z}_{+}$. Its differential is then defined to be the mapping $\mathcal{D} \mathcal{G}\left(u^{0}\right) \in \mathcal{L}_{c}\left(l_{2 e}, l_{2 e}\right)$, where $\mathcal{L}_{c}\left(l_{2 e}, l_{2 e}\right)$ is the space of causal linear mappings from $l_{2 e}$ to $l_{2 e}$, uniquely determined by the condition: $\mathcal{D}\left(\mathcal{P}_{t} \circ \mathcal{G} \circ \mathcal{P}_{t}\right)=\mathcal{P}_{t} \circ \mathcal{D G} \circ \mathcal{P}_{t}$ for all $t \in \mathbb{Z}_{+}$. As a result, if $\mathcal{G}$ is causal and linear, it is differentiable at any point $u^{0} \in l_{2 e}$, and $\mathcal{D} \mathcal{G}\left(u^{0}\right)=\mathcal{G}$. Let $x$ and $w$ be two causal signals belonging to $l_{2 e}$, and $\mathcal{G}\left(q^{-1}\right)$ be a causal operator. If one has : $w=\mathcal{G}\left(q^{-1}\right) x$, then the following relation holds for any $x_{0} \in l_{2 e}$ :

$$
\frac{\partial w}{\partial x}\left(x_{0}\right)=\mathcal{G}\left(q^{-1}\right)
$$

\section{References}

[1] C.A. Desoer, M. Vidyasagar, Feedback systems: Input-Output properties, Academic press, 1975.

[2] A. Feintuch, R. Sacks, Systems theory- A Hilbert Space Approach, Academic Press, 1982.

[3] G.C. Goodwin, K. S. Sin Adaptive filtering prediction and control, Prentice-Hall, 1984.

[4] A. Karimi, I.D. Landau, "Comparison of the closed-loop identification methods in terms of the bias distribution", Systems and control letters, 34(4), 159-167, 1998. 
[5] I.D. Landau, R. Lozano, M. M'Saad, A. Karimi, Adaptive control, second edition, London, Springer, 2011.

[6] I.D. Landau, A.Karimi, "Recursive algorithms for identification in closed-loop: A unified approach and evaluation", Automatica, 33(8), 1499-1523, 1997.

[7] L. Ljung, System identification, theory for the user (second edition), Upper Saddle River, Prentice Hall, 1999.

[8] G. Vinnicombe, "Frequency Domain Uncertainty and the Graph Topology", IEEE Transactions on Automatic Control, 38(9), 1371-1383, 1993.

[9] B.Wahlberg, L.Ljung, "Design variables for bias distribution in transfer function estimation", IEEE transactions on automatic control, 31(2), 134-144, 1986. 